\title{
Stability and Hydration/Dehydration Mechanisms of a Carboxylate-Containing Metal-Organic Framework
}

\author{
Shan Yan, Lina Xu, Hongping Xiao, Xinhua $\mathrm{Li}^{*}$ \\ College of Chemistry and Materials Engineering, Wenzhou University, Wenzhou, China \\ Email: *lixinhua01@126.com
}

How to cite this paper: Yan, S., Xu, L.N., Xiao, H.P. and Li, X.H. (2018) Stability and Hydration/Dehydration Mechanisms of a Carboxylate-Containing Metal-Organic Framework. World Journal of Mechanics, 8, 1-10.

https://doi.org/10.4236/wjm.2018.81001

Received: November 27, 2017

Accepted: January 26, 2018

Published: January 29, 2018

Copyright ( 92018 by authors and Scientific Research Publishing Inc. This work is licensed under the Creative Commons Attribution International License (CC BY 4.0).

http://creativecommons.org/licenses/by/4.0/

\begin{abstract}
Metal-organic frameworks (MOFs) are promising functional compounds; however, they are known to be moisture sensitive. Here, we investigate the hydration and dehydration processes of the carboxylate-containing MOFs $\left[\mathrm{Ni}_{2}(\mathrm{nic})_{4}\left(\mathrm{H}_{2} \mathrm{O}\right)\right]_{\mathrm{n}}$ (nic $=$ nicotinic acid) and the mononuclear complex [Ni(nic) $\left.)_{2}\left(\mathrm{H}_{2} \mathrm{O}\right)_{4}\right]$, respectively. X-ray single crystal diffraction reveals that the hydrogen bonds between water and carboxylic oxygen play a key role in these processes. Molecular-level mechanisms of reversible hydration and dehydration are proposed, based on the competition between water and carboxylic oxygen for coordination with Ni. This study provides important information for future studies on the hydrolytic stability of MOFs in moisture.
\end{abstract}

\section{Keywords}

MOFs, Carboxylate-Containing MOFs, Hydrolytic stability, Hydration and Dehydration Process, Molecular-Level Mechanisms

\section{Introduction}

Owing to their excellent physical and chemical properties, metal-organic frameworks (MOFs) are regarded as promising functional materials with significant application potentials [1] [2] [3]. For instance, IRMOF-1 (MOF-5), IRMOF-3, MIL-53, MIL-101, HKSUT-1, Uio-66, and MOF-74 have exhibited great potentials in storing $\mathrm{H}_{2}$ and $\mathrm{CH}_{4}$ for energy applications [4] [5] [6] [7], heterogeneous catalysis-modified synthesis and metallization [8] [9] [10], luminescent materials [11] [12], magnetic materials [13], and drug delivery [14]. Additionally, MOFs can function as precursors for the synthesis of porous carbon materials [15] [16]. 
Nevertheless, the relatively poor stability of MOFs in moisture has severely limited their industrial applications. Under isolated conditions, they are thermally stable at $350^{\circ} \mathrm{C}-400^{\circ} \mathrm{C}$, which meets the requirements of most applications. However, water adsorption may degrade their structural ordering and stability, as hydration causes the carboxylic groups coordinated to the metallic core to detach in these materials [17] [18] [19].

For this reason, improvements in the hydrolytic stability of MOFs are urgently needed. Indeed, the stability of MOFs in water has been widely studied [20]-[31], and significant progress in structural stability has been achieved by theoretical and experimental means. For instance, the introduction of hydrophobic groups (e.g., $-\mathrm{CH}_{3}$ and $-\mathrm{OCH}_{3}$ ) is an effective way to improve the framework stability of MOFs, because the hydrophobicity and steric hindrance of the substituent groups can separate the coordinatively unsaturated metal ions from water molecules [32] [33] [34]. Therefore, considerable efforts have been made to understand the relationship between the structure of MOFs and their water stability, in order to synthesise more moisture-resistant MOFs [14]. We reported here that hydration of framework complexes is a coordination competition of different oxygen atoms, in which the hydrogen bonds play a key role. This study of framework complex hydration at the molecular level facilitates the understanding of the corresponding mechanisms, and provides good references for improving the hydrothermal stability of framework complexes.

\section{Experimental}

All reagents and chemicals were commercially obtained, and used without further purification.

\subsection{Instrumentation}

The crystal structures were obtained on a Bruker Smart-1000 CCD diffractometer. X-ray powder diffraction (XRPD) measurements were recorded on a Bruker D8 ADVANCE diffractometer at $40 \mathrm{kV}, 40 \mathrm{~mA}$ for $\mathrm{Cu}$ Ka radiation $(\lambda=1.5406$ $\AA$ ), with a step size of $0.01^{\circ}$ in $\theta$ and a scan speed of $0.1 \mathrm{~s}$ per step. Elemental analyses of $\mathrm{C}, \mathrm{H}$ and $\mathrm{N}$ were carried out on a Carlo-Erba 1112 Elemental Analyzer. Infrared (IR) spectra were recorded on an EQUINOX55 spectrophotometer (Bruker) in the range of $4000-400 \mathrm{~cm}^{-1}$ with the $\mathrm{KBr}$ disk technique. Thermogravimetric analysis (TGA) was performed under $\mathrm{N}_{2}$ at a heating rate of 5 ${ }^{\circ} \mathrm{C} /$ min on a TA-Q600 system. A Quantachrome AUTOSORB-MP surface area analyser was used to measure the gas adsorption. The water adsorption/desorption tests were conducted using a high-pressure volumetric analyser (HPVA II, Micromeritics Instrument Ltd., Shanghai, China).

\subsection{Experimental Details}

Synthesis of MOFs $1\left[\mathrm{Ni}_{2}(\mathrm{nic})_{4}\left(\mathrm{H}_{2} \mathrm{O}\right)\right]_{\mathrm{n}}$ (nic = nicotinic acid) was synthesised with hydrothermal method from a mixture of $\mathrm{NiCl}_{2} \cdot 6 \mathrm{H}_{2} \mathrm{O}(1 \mathrm{mmol}, 0.237 \mathrm{~g})$, 
3-cyanopyridine (2 mmol, $0.208 \mathrm{~g}$ ) (The yield and crystallinity of $\left[\mathrm{Ni}_{2}(\mathrm{nic})_{4}\left(\mathrm{H}_{2} \mathrm{O}\right)\right]_{\mathrm{n}}$ can be enhanced by replacing nicotinic acid with 3-cyanopyridine), and $\mathrm{H}_{2} \mathrm{O}$ (20 $\mathrm{mL}$ ) in a $30 \mathrm{~mL}$ Teflon-lined stainless steel reactor. The solution was heated to $150^{\circ} \mathrm{C}$ for $96 \mathrm{~h}$. Then the reaction system was slowly cooled to room temperature. The green block crystals were collected and washed with distilled water and then ethanol (yield: 60\%). Anal. Calcd for $\mathrm{C}_{24} \mathrm{H}_{18} \mathrm{~N}_{4} \mathrm{Ni}_{2} \mathrm{O}_{9}$ (\%) C, 46.21; H, 2.91; N, 8.98. Found: C, 46.16; H, 2.92; N, 9.01. IR (KBr disk, $\left.\mathrm{cm}^{-1}\right)$ : 1637(vs), 1602(s), $1581(\mathrm{~s}), 1576(\mathrm{~m}), 1401(\mathrm{vs}), 1343(\mathrm{~m}), 1316(\mathrm{~m}), 1192(\mathrm{~s}), 1158(\mathrm{~m}), 1091(\mathrm{~m})$, 1057(m), 1046(m), 1035(s), 1101(m), 521(w), 885(w), 821(s), 759(s), 699(s), 643(m), 574(w), 545(w), 451(m), and $440(\mathrm{~m}) \mathrm{cm}^{-1}$.

Transformation of the MOFs 1 crystal to the mononuclear complex 2, ([Ni(nic) $\left.\left.)_{2}\left(\mathrm{H}_{2} \mathrm{O}\right)_{4}\right]\right)$. The 3D framework structure of 1 was first reported by $\mathrm{Wu}$ [35], but the sorption of water following its hydration was not noted. MOFs 1 can transform to 2 [36] completely at room temperature in atmosphere [37]. When 1 was heated in water, the transformation process is accelerated. Anal. Calcd for $\mathrm{C}_{12} \mathrm{H}_{16} \mathrm{~N}_{2} \mathrm{NiO}_{8}$ (\%) C, 38.44; $\mathrm{H}, 4.30$; N, 7.47. Found: C, 38.46; $\mathrm{H}, 4.26$; $\mathrm{N}, 7.51$. IR ( $\mathrm{KBr}$ disk, $\left.\mathrm{cm}^{-1}\right)$ : 1618(vs), 1575(vs), 1393(s), 1198(m), 1112(m), 1050(m), 842(m), 765(s), 686(w), and 600(w) $\mathrm{cm}^{-1}$.

\section{Results and Discussions}

Although the structures of 1 and $\mathbf{2}$ have been reported previously [35] [36], the interconversion between them via dehydration/hydration processes has not been fully understood [37]. According to previous studies [35], 1 is a green block crystal with a 3D mushroom-like crystal structure, and it can be completely converted to the pale blue powder of 2 when exposed to air for about 60 days. The transition from 1 to 2 is through a hydration process. The XRPD pattern of the blue powder has been reported [37] [38], and the simulated pattern is based on the atomic coordinates of $\mathbf{2}$ [36] (Supplementary Information).

Our experiments revealed that 1 and 2 can be converted into each other: the hydration of 1 in air or a heated solution resulted in $\mathbf{2}$ (with significantly higher rates in the heated solution), and the dehydration of 2 under hydrothermal conditions resulted in 1 (Supplementary Information). The nickel ions in 1 were sixcoordinated by two nitrogen atoms and four carboxylic oxygen atoms (Figure 1 ), while in 2 they were six-coordinated (Figure 2, beige octahedron) by two nitrogen atoms and four water molecules $(\mathrm{O} 1-\mathrm{O} 4)$. Therefore, the coordinated carboxylic oxygen atoms in 1 were substituted by water molecules during hydration. The bidentate coordination of bridging water molecules was changed into monodentate coordination during hydration to produce 2 . In this process, seven water molecules were required for every two molecules of 2 . In the reverse process of dehydration under hydrothermal conditions, these water molecules were substituted by carboxylic oxygen atoms. Hence, it can be concluded that water molecules and carboxylic groups compete with each other for nickel coordination, and the hydration and dehydration reactions are reversible. 


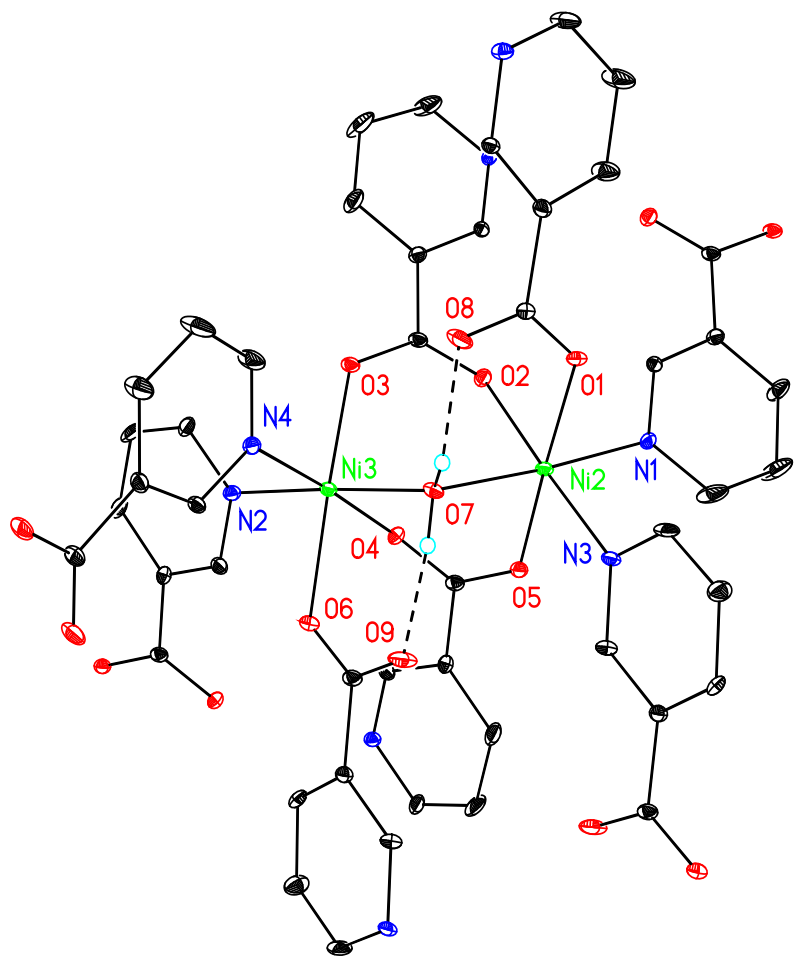

Figure 1. ORTEP drawing with labelling scheme for compound $1\left[\mathrm{Ni}_{2}(\mathrm{nic})_{4}\left(\mathrm{H}_{2} \mathrm{O}\right)\right]_{\mathrm{n}}$; some $\mathrm{H}$ atoms are omitted for clarity.

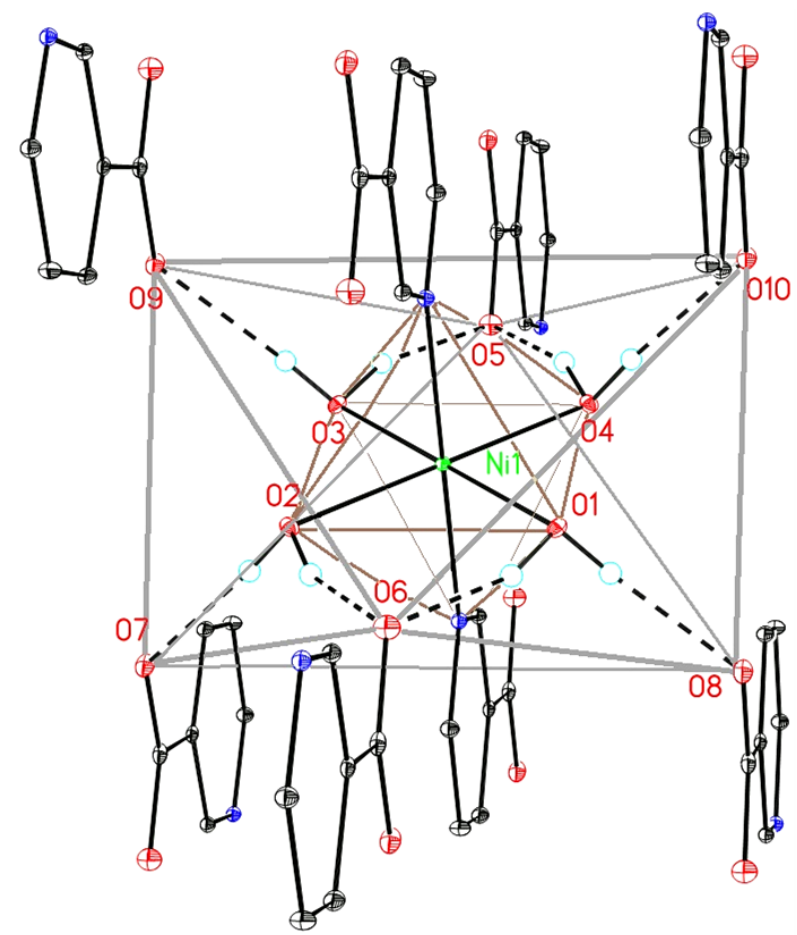

Figure 2. ORTEP drawing with labelling scheme for compound $2\left[\mathrm{Ni}(\mathrm{nic})_{2}\left(\mathrm{H}_{2} \mathrm{O}\right)_{4}\right]$, some atoms are omitted for clarity. Beige: coordinated octahedron, grey: uncoordinated octahedron. 
According to the structure of 2 (Figure 2), the six-coordinated Nil ion was surrounded by six other 2 molecules (O5-O10, which formed the grey octahedron); each of them contained an uncoordinated carboxylic oxygen atom linked to a coordinated water molecule via hydrogen bonds. Therefore, the Ni1 ion was surrounded by a coordinated octahedron of four water molecules $(\mathrm{O} 1-\mathrm{O} 4)$ and two $\mathrm{N}$ atoms, and an uncoordinated octahedron of 2 molecules connected by hydrogen bonds. These two octahedrons intersect each other spatially to minimise the repulsive force. In the uncoordinated octahedron, the axial atoms $\mathrm{O} 5$ and $\mathrm{O} 6$ were each located between and connected to two coordinated water molecules in the same plane. Meanwhile, the four carboxylic oxygen atoms above and below this plane (O7-O10) were located between the coordinated water molecules and nitrogen atoms, and linked to the water molecules via individual hydrogen bonds. In this way, the equatorial plane of the uncoordinated octahedron was established.

Based on the above spatial configuration, we concluded that the two octahedrons competed against each other for coordinating with Ni1. Under ambient conditions, the carboxylic oxygen atoms were located far from Ni1 due to steric hindrance effects, and 2 was favoured over 1 in terms of stability. The coordinated octahedrons in 1 were squeezed, while those in 2 were stretched which were more stable due to the Jahn-Teller effect.

On the other hand, under hydrothermal conditions with high temperature and pressure, the bulky carboxylic oxygen atoms were more effective than the small water molecules at coordination. The two coordinated nitrogen atoms were not substituted by oxygen atoms, due to their high coordination affinity. Because of steric hindrance, one coordinated carboxylic group of the niacin was substituted by a small water molecule (O7 in Figure 1), which acted as a bidentate ligand that connects two adjacent $\mathrm{Ni1}$ ions to form a dimer structure. In this way, Ni was coordinatively saturated and compound 1 was obtained. Note that the nitrogen atoms, although still coordinated, were shifted from their positions in 2 by the repulsive force.

From the TGA result, guest water was not observed in the pores of the $3 \mathrm{D}$ porous structure of 1 . Only two intra-molecular hydrogen bonds would be formed between the uncoordinated carboxylic oxygen atoms and the hydrogen atoms in the coordinated water molecules. As shown in the stacked graph (Figure 3), the presence of mushroom-shaped pores in 1 could severely limit the free space in the vicinity of $\mathrm{Ni1}$, and no uncoordinated octahedrons were allowed. Within these pores, up to four water molecules localized by hydrogen bonds were allotted the free space between two coordinated carboxylic oxygen atoms, specifically.

The maximum quantity of moisture adsorption observed was $8.44 \%$ (Figure 4 ), which is equivalent to three water molecules per Ni1. This is close to the theoretical prediction of four water molecules per Nil, and we attribute the difference to experimental errors. The hydration was expected to occur in multiple steps, and as many as seven water molecules were required for each 1 molecule. 


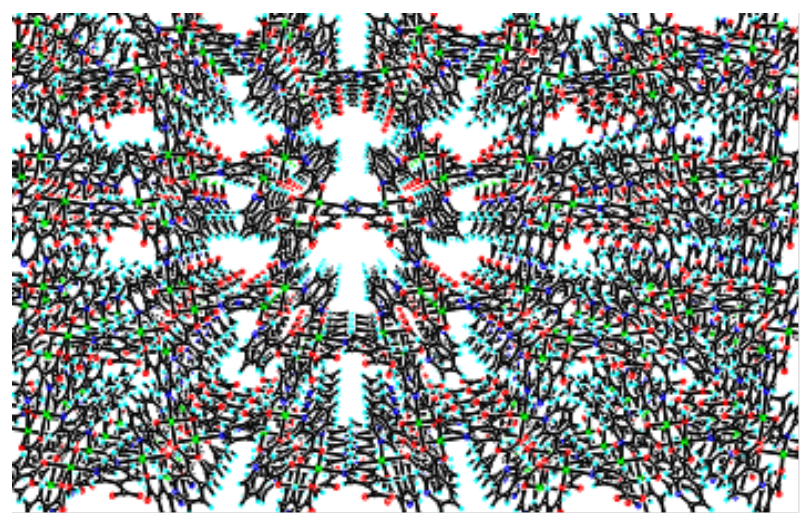

Figure 3. 3D structure of 1 , showing the mushroom-shaped pores.

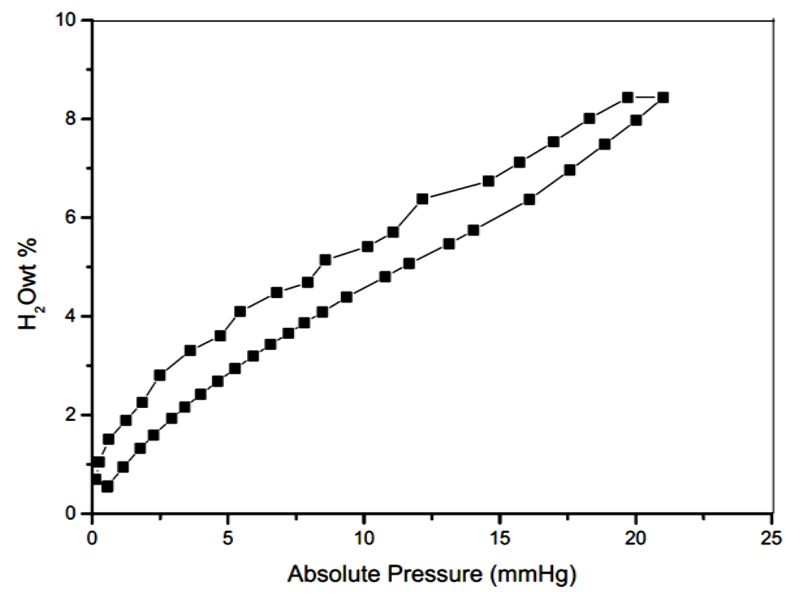

Figure 4. Shows the isothermal moisture adsorption/desorption curve of 1 .

Therefore, the extra water molecules must come from adsorption. Specifically, the 3D structure of 1 was destroyed when one coordinated carboxylic oxygen was substituted by water, creating more free space in the vicinity of Ni1. This enabled external water molecules to enter this space to further hydrolyse the carboxylic groups, resulting in 2. This multi-step mechanism may explain the extremely slow hydration rate of 1 under ambient conditions. As mentioned earlier, the coordinated nitrogen atoms were not substituted by water, due to their higher coordination affinity. Water molecules that were localized by hydrogen bonds generated another ligand field in the vicinity of Nil. Due to their small size, these water molecules were close to Nil to readily substitute the carboxylic groups under ambient instead of hydrothermal condition. This also serves as an explanation to the moisture sensitivity of MOFs. By comparison, in all N-coordinated MOFs such accumulation of water molecules in the vicinity of metal ions and additional ligand fields have not been observed, as no hydrogen bonds could be established due to the high alkalinity of these MOFs. Hence, the N-coordinated MOFs exhibited excellent water stability.

In the solution phase, the water molecules could be dispersed without estab- 
lishing the additional ligand fields. In real solutions, however, water molecules were nevertheless localized in the vicinity of metal ions by the hydrogen bonds, resulting in a local micro-environment in which the substitution of carboxylic groups by water molecules was favoured.

In summary, we propose that water adsorption on 1 is a dynamic process based on crystal structure change; and the hydration of 1 is achieved in multiple steps in which the hydrogen bonds play a key role. The two coordinated nitrogen atoms are not substituted during the conversion between 1 and 2 , although their locations are shifted.

\section{Conclusions}

In the dehydrated MOFs $\left[\mathrm{Ni}_{2}(\mathrm{nic})_{4}\left(\mathrm{H}_{2} \mathrm{O}\right)\right]_{\mathrm{n}} 1$, the adsorbed water molecules were localized in the vicinity of the metal ions, due to the hydrogen bonds between the coordinated water molecules and carboxylic groups (since other parts of the structure were hydrophobic). Located in the free space within the octahedron of coordinated water molecules and carboxylic groups, the adsorbed water molecules generated another ligand field (at an angle to the first octahedron) that interacted with the $\mathrm{Ni}$ ion and competed with the original oxygen ligands. Under ambient conditions, the adsorbed water molecules exhibited higher affinity to the metal ions due to their proximity. As a result, the MOFs were hydrolysed. The nitrogen atoms were not substituted due to their high coordination affinity.

Similarly, in the mononuclear complex 2 , an accumulation of uncoordinated carboxylic groups in the vicinity of the metal ions were also observed, which can be attributed to hydrogen bonds. Located in the free space within the octahedron of coordinated water molecules, these uncoordinated carboxylic groups formed another octahedron and competed with the coordinated octahedron. Under high temperatures and pressures, the carboxylic oxygen atoms exhibited higher coordination affinity than water, and the reversible dehydration of $\mathbf{2}$ was favoured. In essence, we found that the hydration of MOFs occurred by the coordination substitution of oxygen atoms, in which hydrogen bonds played a key role. This study of MOFs hydration at the molecular level will facilitate the understanding of hydration mechanism of frameworks, and provide important information for improving the hydrothermal stability of MOFs.

\section{Acknowledgements}

We gratefully acknowledge the financial support provided by the National Natural Science Foundation of China (Grant nos. 21571144), the Natural Science Foundation of Zhejiang Province (No. LQ15B030001), and Faculty of Chemistry \& Material Engineering, Wenzhou University.

\section{References}

[1] Canivet, J., Fateeva, A., Guo, Y., Coasnecd, B. and Farrusseng, D. (2014) Water Adsorption in MOFs: Fundamentals and Applications. Chemical Society Reviews, 43, 5594-5617. https://doi.org/10.1039/C4CS00078A 
[2] Ferey, G., Mellot-Draznieks, C., Serre, C. and Millange, F. (2005) Crystallized Frameworks with Giant Pores: Are There Limits to the Possible? Accounts of Chemical Research, 38, 217-225. https://doi.org/10.1021/ar040163i

[3] Zhu, Q.L. and Xu, Q. (2014) Metal-Organic Framework Composites. Chemical Society Reviews, 43, 5468-5512. https://doi.org/10.1039/C3CS60472A

[4] Xuan, W., Zhu, C., Liu, Y. and Cui, Y. (2012) Mesoporous Metal-Organic Framework Materials. Chemical Society Reviews, 41, 1677-1695. https://doi.org/10.1039/C1CS15196G

[5] Furukawa, H., Ko, N., Go, Y.B., Aratani, N., Choi, S.B., Choi, E., Yazaydin, A.Ö., Snurr, R.Q., O'Keeffe, M., Kim, J. and Yaghi, O.M. (2010) Ultrahigh Porosity in Metal-Organic Frameworks. Science, 329, 424-428.

https://doi.org/10.1126/science.1192160

[6] Suh, M.P., Park, H.J., Prasad, T.K. and Lim, D.W. (2012) Hydrogen Storage in Metal-Organic Frameworks. Chemical Reviews, 112, 782-835.

https://doi.org/10.1021/cr200274s

[7] Kitagawa, S., Kitaura, R. and Noro, S.I. (2004) Functional Porous Coordination Polymers. Angewandte Chemie International Edition, 43, 2334-2375. https://doi.org/10.1002/anie.200300610

[8] Chughtai, A.H., Ahmad, N., Younus, H.A., Laypkovc, A. and Verpoort, F. (2015) Metal-Organic Frameworks: Versatile Heterogeneous Catalysts for Efficient Catalytic Organic Transformations. Chemical Society Reviews, 44, 6804-6849. https://doi.org/10.1039/C4CS00395K

[9] Wong, A.G., Matzger, A.J. and Yaghi, O.M. (2006) Exceptional $\mathrm{H}_{2}$ Saturation Uptake in Microporous Metal-Organic Frameworks. Journal of the American Chemical Society, 128, 3494-3495. https://doi.org/10.1021/ja058213h

[10] Hafizovic, J., Bjorgen, M., Olsbye, U., Dietzel, P.D.C., Bordiga, S., Prestipino, C., Lamberti, C. and Lillerud, K.P. (2007) The Inconsistency in Adsorption Properties and Powder XRD Data of MOF-5 Is Rationalized by Framework Interpenetration and the Presence of Organic and Inorganic Species in the Nanocavities. Journal of the American Chemical Society, 129, 3612-3620. https://doi.org/10.1021/ja0675447

[11] Dang, S., Zhang, J.H. and Sun, Z.M. (2012) Tunable Emission Based on Lanthanide(III) Metal-Organic Frameworks: An Alternative Approach to White Light. Journal of Materials Chemistry, 22, 8868-8873. https://doi.org/10.1039/c2jm30389b

[12] Cabria, I., Lopez, M.J. and Alonso, J.A. (2008) Shape of the Hydrogen Adsorption Regions of MOF-5 and Its Impact on the Hydrogen Storage Capacity. Physical Review B, 78, 205432. https://doi.org/10.1103/PhysRevB.78.205432

[13] Kurmoo, M. (2009) Magnetic Metal-Organic Frameworks. Chemical Society Reviews, 38, 1353-1379. https://doi.org/10.1039/b804757j

[14] Horcajada, P., Gref, R., Baati, T., Allan, P.K., Maurin, G., Couvreur, P., Férey, G., Morris, R.E. and Serre, C. (2012) Metal-Organic Frameworks in Biomedicine. Chemical Reviews, 112, 1232-1268. https://doi.org/10.1021/cr200256v

[15] Zhang, J., Yang, W., Wu, X.Y., Zhang, L. and Lu, C.Z. (2016) Synthesis, Photoluminescence, and Gas Adsorption Properties of a New Furan-Functionalized MOF and Direct Carbonization for Synthesis of Porous Carbon. Crystal Growth and Design, 16, 475-482. https://doi.org/10.1021/acs.cgd.5b01481

[16] Chaikittisilp, W., Hu, M., Wang, H., Huang, H.S., Fujita, T., Wu, K.C.W., Chen, L.C., Yamauchi, Y. and Ariga, K. (2012) Nanoporous Carbons through Direct Carbonization of a Zeolitic Imidazolate Framework for Supercapacitor Electrodes. 
Chemical Communications, 48, 7259-7261. https://doi.org/10.1039/c2cc33433j

[17] Tan, K., Nijem, N., Gao, Y., Zuluaga, S., Li, J., Thonhauserb, T. and Chabal, Y.J. (2015) Water Interactions in Metal Organic Frameworks. CrystEngComm, 17, 247-260. https://doi.org/10.1039/C4CE01406E

[18] Grajciar, L., Bludsky, O. and Nachtigall, P. (2010) Water Adsorption on Coordinatively Unsaturated Sites in CuBTC MOF. The Journal of Physical Chemistry Letters, 1, 3354-3359. https://doi.org/10.1021/jz101378z

[19] Moghadam, P.Z., Fairen-Jimenez, D. and Snurr, R.Q. (2016) Efficient Identification of Hydrophobic MOFs: Application in the Capture of Toxic Industrial Chemicals. Journal of Materials Chemistry A, 4, 529-536. https://doi.org/10.1039/C5TA06472D

[20] Long, J.R. and Yaghi, O.M. (2009) The Pervasive Chemistry of Metal-Organic Frameworks. Chemical Society Reviews, 38, 1213-1214. https://doi.org/10.1039/b903811f

[21] Jasuja, H. and Walton, K.S. (2013) Effect of Catenation and Basicity of Pillared Ligands on the Water Stability of MOFs. Dalton Transactions, 42, 15421-15426. https://doi.org/10.1039/c3dt51819a

[22] Li, J.R., Sculley, J. and Zhou, H.C. (2012) Metal-Organic Frameworks for Separations. Chemical Reviews, 112, 869-932. https://doi.org/10.1021/cr200190s

[23] Fernandez, C.A., Nune, S.K., Annapureddy, H.V., Dang, L.X., McGrail, B.P., Zheng, F., Polikarpov, E., King, D.L., Freeman, C. and Brooks, K.P. (2015) Hydrophobic and Moisture-Stable Metal-Organic Frameworks. Dalton Transactions, 44, 13490-13497. https://doi.org/10.1039/C5DT00606F

[24] DeCoste, J.B., Peterson, G.W., Jasuja, H., Glover, T.G., Huang, Y.G. and Walton, K.S. (2013) Stability and Degradation Mechanisms of Metal-Organic Frameworks Containing the $\mathrm{Zr}_{6} \mathrm{O}_{4}(\mathrm{OH})_{4}$ Secondary Building Unit. Journal of Materials Chemistry $A, 1,5642-5650$. https://doi.org/10.1039/c3ta10662d

[25] DeCoste, J.B., Peterson, G.W., Schindler, B.J., Killops, K.L., Broweb, M.A. and Mahle, J.J. (2013) The Effect of Water Adsorption on the Structure of the Carboxylate Containing Metal-Organic Frameworks Cu-BTC, Mg-MOF-74, and UiO-66. Journal of Materials Chemistry A, 1, 11922-11932. https://doi.org/10.1039/c3ta12497e

[26] Gelfand, B.S. and Shimizu, G.K.H. (2016) Parameterizing and Grading Hydrolytic Stability in Metal-Organic Frameworks. Dalton Transactions, 45, 3668-3678. https://doi.org/10.1039/C5DT04049C

[27] Ahrenholtz, S.R., Epley, C.C. and Morris, A.J. (2014) Solvothermal Preparation of an Electrocatalytic Metalloporphyrin MOF Thin Film and its Redox Hopping Charge-Transfer Mechanism. Journal of the American Chemical Society, 136, 2464-2472. https://doi.org/10.1021/ja410684q

[28] Han, S. and Lah, M.S. (2015) Simple and Efficient Regeneration of MOF-5 and HKUST-1 via Acid-Base Treatment. Crystal Growth and Design, 15, 5568-5572. https://doi.org/10.1021/acs.cgd.5b01218

[29] Sumida, K., Rogow, D.L., Mason, J.A., McDonald, T.M., Bloch, E.D., Herm, Z.R., Bae, T.H. and Long, J.R. (2012) Carbon Dioxide Capture in Metal-Organic Frameworks. Chemical Reviews, 112, 724-781. https://doi.org/10.1021/cr2003272

[30] Corma, A., Garcia, H. and Xamena, F.X.L. (2010) Engineering Metal Organic Frameworks for Heterogeneous Catalysis. Chemical Reviews, 110, 4606-4655. https://doi.org/10.1021/cr9003924

[31] Yoon, M., Srirambalaji, R. and Kim, K. (2012) Homochiral Metal-Organic Frame- 
works for Asymmetric Heterogeneous Catalysis. Chemical Reviews, 112, 1196-1231. https://doi.org/10.1021/cr2003147

[32] Li, H., Eddaoudi M., O’Keeffe, M. and Yaghi, O.M. (1999) Design and Synthesis of an Exceptionally Stable and Highly Porous Metal-Organic Framework. Nature, 402, 276-279. https://doi.org/10.1038/46248

[33] Chui, S.S.Y., Los, S.M.F., Charmant, J.P.H. and Williams, I.D. (1999) A Chemically Functionalizable Nanoporous Material $\left[\mathrm{Co}_{3}(\mathrm{TMA})_{2}\left(\mathrm{H}_{2} \mathrm{O}\right)_{3}\right]_{n}$. Science, 283, 1148-1150. https://doi.org/10.1126/science.283.5405.1148

[34] Kathalikkattil, A.C., Roshan, R., Tharun, J., Babu, R., Jeong, G.S., Kim, D.W., Choc, S.J. and Park, D.W. (2016) A Sustainable Protocol for the Facile Synthesis of Zinc-Glutamate MOF: An Efficient Catalyst for Room Temperature $\mathrm{CO}_{2}$ Fixation Reactions under Wet Conditions. Chemical Communications, 52, 280-283. https://doi.org/10.1039/C5CC07781H

[35] Wu, C.D., Lu, C.Z., Zhuang, H.H. and Huang, J.S. (2003) Structure and Magnetic Property of a New 3D Nicotinic Acid Bridged Nickel Polymer. Zeitschrift fur anorganische und allgemeine Chemie, 629, 693-696.

https://doi.org/10.1002/zaac.200390117

[36] Batten, S.R. and Harris, A.R. (2001) Trans-Tetra-Aqua-Bis-(Pyridine-3-Carboxyl-

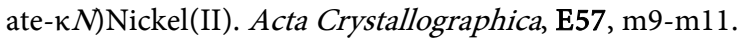
https://doi.org/10.1107/S1600536800018742

[37] Huang, X., Jiang, L.J., Hu, M.L. and Li, X.H. (2011) A dynamically Nanoporous Metal-Organic Framework Functional Properties. Advanced Materials Research, 239-242, 3150-3155. https://doi.org/10.4028/www.scientific.net/AMR.239-242.3150

[38] Anagnostopoulos, A., Matthews, R.W., Walton, R.A. (1972) Studies on Metal Carboxylates. Part II. Cobalt(II) and Nickel(II) Complexes of Certain Pyridine Carboxylic Acids and Pyridine-3-Sulfonic Acid: Magnetic and Spectral Studies. Canadian Journal of Chemical Engineering, 50, 1307-1314. https://doi.org/10.1139/v72-205 